\title{
Host-antibody inductivity of virulent Entamoeba histolytica and non-virulent Entamoeba moshkovskii in a mouse model
}

\author{
Narumol Khomkhum', Somphob Leetachewa ${ }^{2}$, Aulia Rahmi Pawestri ${ }^{1}$ and Saengduen Moonsom ${ }^{*}$
}

\begin{abstract}
Background: Despite similarities in morphology, gene and protein profiles, Entamoeba histolytica and E. moshkovskii show profound differences in pathogenicity. Entamoeba histolytica infection might result in amoebic dysentery and liver abscess, while E. moshkovskii causes only mild diarrhea. Extensive studies focus on roles of host immune responses to the pathogenic E. histolytica; however, evidence for E. moshkovskii remains scarce.

Methods: To study differences in host-antibody response profiles between E. histolytica and E. moshkovskii, mice were immunized intraperitoneally with different sets of Entamoeba trophozoites as single species, mixed species and combinations.

Results: Mice prime-immunized with E. histolytica and E. moshkovskii combination, followed by individual species, exhibited higher lgG level than the single species immunization. Mice immunized with E. moshkovskii induced significantly higher levels and long-lasting antibody responses than those challenged with E. histolytica alone. Interestingly, E. histolytica-specific anti-sera promoted the cytopathic ability of E. histolytica toward Chinese hamster ovarian $(\mathrm{CHO})$ cells, but showed no effect on cell adhesion. There was no significant effect of immunized sera on cytopathic activity and adhesion of E. moshkovskii toward both $\mathrm{CHO}$ and human epithelial human colonic (Caco-2) cell lines. Monoclonal-antibody (mAb) characterization demonstrated that $89 \%$ of E. histolytica-specific mAbs produced from mice targeted cytoplasmic and cytoskeletal proteins, whereas $73 \%$ of E. moshkovskii-specific mAbs targeted plasma membrane proteins.
\end{abstract}

Conclusions: The present findings suggest that infection with mixed Entamoeba species or E. moshkovskii effectively induces an antibody response in mice. It also sheds light on roles of host antibody response in the pathogenic difference of E. histolytica and E. moshkovskii trophozoites, and cell surface protein modifications of the amoebic parasites to escape from host immune system.

Keywords: Amoebiasis, Entamoeba histolytica, Entamoeba moshkovskii, Pathogenicity, Antibody dependent enhancement

\section{Background}

Entamoeba histolytica is an anaerobic pathogenic protozoan parasite that causes approximately 100,000 global deaths annually due to amoebiasis [1]. Disease symptoms range from mild diarrhea to severe bloody diarrhea with mucus as the parasite invades the intestinal epithelium

\footnotetext{
*Correspondence: saengduen.moo@mahidol.ac.th

${ }^{1}$ Department of Protozoology, Faculty of Tropical Medicine, Mahidol

University, Ratchawithi, Bangkok 10400, Thailand

Full list of author information is available at the end of the article
}

[2]. After invading the intestinal lamina propria, this parasite enters the blood stream to reach other organs and results in abscesses, most commonly in the liver [3], and rarely in lungs [4] and brain [5]. The pathogenesis of $E$. histolytica starts with parasite adhesion at the large intestinal epithelium and secretion of cysteine proteases, leading to the degradation of host tissues. The secreted cysteine proteases play important roles in degrading gut mucosal IgA and circulating IgG, resulting in the ineffectiveness or failure of host immunity, thus inversely 
promote extra-intestinal infection of E. histolytica [6, 7]. In addition, the parasite-gut adhesion was shown to trigger host signal transductions through caspases 3-like cascade and caspases 8- and 9-independent manner [8]. These lead to apoptotic cell death, which were preferentially phagocytosed by the parasite. The interaction also stimulates production of pro-inflammatory cytokines, including interleukin (IL)-1, IL-6, IL-8, IFN- $\gamma$ and tumor necrosis factor (TNF)- $\alpha$, which consequentially promote tissue damages and severity of the disease $[9,10]$. Inhibition of TNF- $\alpha$ has been proved to significantly reduce the inflammation and tissue destruction [11], while the absence of the anti-inflammatory cytokine IL-10 has been shown to result in increased severity of intestinal amoebiasis [12]. Thus, the manifestation of amoebiasis apparently happens through the parasite's ability to activate cytokine-mediated cell deaths and manipulate the host immune system.

Entamoeba moshkovskii was previously considered as a non-pathogenic protozoan parasite, which was commonly found to co-occur in human stools collected from $E$. histolytica endemic areas, often leading to misdiagnosis of $E$. histolytica due to their mostly identical morphology [13, 14]. Despite being considered non-pathogenic, E. moshkovskii has been gradually reported as associated with diarrhea in humans and mice [15-17]. Recently, E. moshkovskii was reported to cause subcutaneous abscess in Indonesia [18]. Shimokawa et al. [16] showed that E. moshkovskii was able to cause symptoms, including weight loss, diarrhea and colitis in susceptible mice as is the case for E. histolytica. Furthermore, damages of the intestinal epithelium of $E$. moshkovskii-infected mice were observed due to host IFN- $\gamma$ mediated cell apoptosis [17].

In the present study, we aimed to investigate the immunogenicity of E. histolytica and E. moshkovskii trophozoites through host-antibody response profiles as well as effect of the immunized sera on Entamoeba pathogenicity. We found that mouse immunization with mixed Entamoeba species was able to induce both specific IgA and IgG higher levels than single species. The effect of the immunized sera on cytopathic activity and host cell adhesion were investigated and the possible immune evasion and cell manipulating mechanisms by $E$. histolytica are discussed. Our findings may shed more light on $E$. histolytica pathogenicity, which can be of further benefit in the development of diagnosis modalities, treatment and vaccines for this parasite.

\section{Methods}

Mouse immunization with Entamoeba cells

Trophozoite cells of E. histolytica strain HM1: IMSS and

E. moshkovskii strain Laredo, which were kindly provided by Professor Tomoyoshi Nozaki, Department of Biomedical Chemistry, Graduate School of Medicine, University of Tokyo, Japan, were axenically cultured in bis-iron serum (BIS) medium at $37{ }^{\circ} \mathrm{C}$ and $26.5^{\circ} \mathrm{C}$, respectively. Cells were harvested by placing culture tubes on ice for 10 min to detach the cells, followed by centrifugation at $200 \times g$ for $3 \mathrm{~min}$ at $4{ }^{\circ} \mathrm{C}$ with three washes using cold phosphate-buffered saline (PBS). Viable amoeba cells were counted using a hemocytometer by trypan blue exclusion ( $0.2 \%$ trypan blue). For studies of host-antibody response, $\mathrm{BALB} / \mathrm{c}$ mice (3 mice/group; 12 mice in total) were immunized with $2 \times 10^{6}$ cells of mixed species $\left(1 \times 10^{6}\right.$ cells each of E. histolytica and E. moshkovskii) or $2 \times 10^{6}$ cells of individual species (E. histolytica or E. moshkovskii) according to 4 immunization designs (group 1 mice received E. histolytica cells for 4 doses; group 2 mice received E. moshkovskii cells for 4 doses; group 3 mice received $E$. histolytica and $E$. moshkovskii cell mixture for 2 doses, followed by E. histolytica cells for 2 doses; group 4 mice received $E$. histolytica and $E$. moshkovskii cell mixture for 2 doses followed by $E$. moshkovskii cells for 2 doses). Immunization was performed intraperitoneally (IP) with two-week intervals. Whole blood was collected from the ventral tail vein before each immunization [19] and after the 4th boost for 2 weeks (B4: bleed 4) and 8 weeks (B5: bleed 5). Serum collected before the first immunization (pre-immunized serum) was used as a negative control for the baseline antibody level of each mouse.

\section{Monoclonal antibody (mAb) production}

BALB/c mice (2 mice per set) were immunized with $2 \times 10^{6}$ cells of E. histolytica and E. moshkovskii trophozoite for 3 doses followed by 2 doses of mixed Entamoeba cells $\left(1 \times 10^{6}\right.$ cells each of E. histolytica and E. moshkovskii) in two-week intervals. Blood samples were collected from the tail vein before each immunization and tested for Entamoeba-specific antibodies. The mice were sacrificed and splenic B cells were fused with mouse myeloma cells using the standard hybridoma technique described by Moonsom et al. [19]. Hybridoma cells secreting Entamoeba-specific antibodies were screened by enzymelinked immunosorbent assay (ELISA) with Entamoeba cell lysate proteins. Limiting dilutions were performed to obtain the mAb-producing cells.

\section{Preparation of cellular protein compartments}

Axenically cultivated E. histolytica and E. moshkovskii trophozoite cells were harvested and washed with PBS, $\mathrm{pH}$ 7.4. Total cell lysate proteins were solubilized using mammalian protein extraction reagent (M-PER) (Thermo Fisher Scientific, Waltham, MA, USA). Cells were fractioned into cytoplasmic, membranous, nuclear and 
cytoskeletal portions using a Qproteome cell compartment kit (Qiagen, Hilden, Germany) following the manufacturer's instructions. Protein profiles of cell fractions were analyzed on a $10 \%$-gel using sodium dodecyl sulfate-polyacrylamide gel electrophoresis (SDS-PAGE) and protein concentration was determined using Bradford's assay following the manufacturer's instructions (Bio-Rad, Hercules, CA, USA).

\section{ELISA of anti-serum and mAbs with cellular fractions}

Cellular protein fractions were immobilized onto the ELISA microplate for overnight at $4{ }^{\circ} \mathrm{C}$. After blocking with $2 \%$ skim milk, culture supernatant or the immunized mouse serum containing primary antibodies; anti-E. histolytica (anti-Eh4), anti-E. moshkovskii (antiEm6) and anti-pan-human Entamoeba; E. histolytica, E. moshkovskii and E. dispar (anti-Ehmd4) mAb or 1:5000 diluted pre-immunized serum (a control) were applied. The reactions were incubated for $1 \mathrm{~h}$ at room temperature. Entamoeba binding partner-mAb complexes were detected with 1:5000 diluted anti-mouse immunoglobulins (Thermo Fisher Scientific), anti-mouse IgG (SigmaAldrich, St. Louis, MO, USA) and anti-mouse IgA (Sigma-Aldrich) conjugated with horse radish peroxidase (HRP) enzyme (Thermo Fisher Scientific) for $1 \mathrm{~h}$. The colorimetric signal was developed using 3,3',5,5'-tetramethylbenzidine (TMB) substrate (Thermo Fisher Scientific) and quenched with $1 \mathrm{~N}$ hydrochloric acid $(\mathrm{HCl})$. The color intensity was recorded at a wavelength of $450 \mathrm{~nm}$ using a Sunrise microplate reader (Tecan, Männedorf, Switzerland). The measurement was performed in triplicate and presented as means of optical density (OD) \pm standard error of the mean (SEM).

\section{Immunofluorescent assay of specific anti-sera with Entamoeba cells}

Axenically cultivated trophozoites of E. histolytica and E. moshkovskii were harvested and attached onto cell imaging slides (Eppendorf, Hamburg, Germany). The immobilized cells were fixed with $3.7 \%$ paraformaldehyde at room temperature for $10 \mathrm{~min}$ and permeabilized with $0.2 \%$ Triton $\mathrm{X}-100$ in $1 \%$ bovine serum albumin (BSA)PBS for $10 \mathrm{~min}$. Non-permeabilized (incubated in 1\% BSA-PBS) and permeabilized cells were reacted with the immunized mouse serum (1:1000) or culture supernatant containing $\mathrm{mAb}(1 \mathrm{mg} / \mathrm{ml} \mathrm{mAb})$ for $1 \mathrm{~h}$. Antibody positive cells were detected with anti-mouse immunoglobulin G conjugated with FITC (1:60) and viewed by confocal microscopy (LSM 700; Carl Zeiss, Jena, Germany). All immunological reactions were performed in $1 \%$ BSA-PBS.

\section{$\mathrm{CHO}$ cytopathic and cell-adhesion assays of $E$. histolytica and E. moshkovskii}

Chinese hamster ovary $(\mathrm{CHO})$ cell lines were cultured in Roswell Park Memorial Institute (RPMI) medium supplemented with $10 \%$ fetal bovine serum (FBS) at $37{ }^{\circ} \mathrm{C}$ in a $\mathrm{CO}_{2}$ incubator. Cells were harvested by $0.1 \mathrm{mM}$ ethylene diamine tetraacetic acid (EDTA)-PBS, pH 7.4 and washed 3 times with cold PBS. Viable trophozoite cells of E. histolytica $\left(1 \times 10^{5}\right.$ cells) and E. moshkovskii $\left(2 \times 10^{5}\right.$ cells) were pre-incubated with pre-immunized or immunized serum (1:5000 dilution in 10\% FBS-RPMI medium) at $4{ }^{\circ} \mathrm{C}$ for $30 \mathrm{~min}$. The Entamoeba cell-serum mixtures were then incubated with $2 \times 10^{5}$ of $\mathrm{CHO}$-cell suspension at a ratio of 1:5 for Entamoeba and $\mathrm{CHO}$ cells at $4{ }^{\circ} \mathrm{C}$ for an additional $1 \mathrm{~h}$. CHO cells incubated with Entamoeba cells in culture medium or serum alone were used as controls. CHO cell adhesion was defined as an Entamoeba cell formed with at least three $\mathrm{CHO}$ cells $[20,21]$. CHOEntamoeba cell mixtures were stained with $500 \mathrm{nM}$ propidium iodine (PI). PI positive $\mathrm{CHO}$ cells were counted under fluorescence microscopy (Observer Z1; Carl Zeiss) to assess the killing ability of Entamoeba cells. The assay was performed in triplicate and presented as percent amoeba cell adhesion \pm standard error of the mean (SEM) or percent cell death with $95 \%$ confidence interval (CI).

\section{Entamoeba histolytica and E. moshkovskii adhesion to human epithelial Caco-2 cell line}

Entamoeba histolytica or E. moshkovskii cells $\left(2 \times 10^{5}\right)$ were pre-mixed separately with a pre-immunized and dose-4 immunized serum at a final dilution of 1:5000 in $250 \mu \mathrm{l}$ of $10 \%$ FCS-Iscove's Modified Dulbecco's Medium (IMDM). The Entamoeba cell-serum mixture was incubated with a monolayer of Caco- 2 cells (provided by Professor Kris Chadee, Department of Microbiology, Immunology and Infectious Diseases, University of Calgary, Canada) in a 24-well plate at the ratio of $1: 1$ of Entamoeba:Caco- 2 cells at $37{ }^{\circ} \mathrm{C}$ for $15 \mathrm{~min}$. The culture supernatant was removed and then the cells were washed twice with PBS. The adhered E. histolytica/E. moshkovskii cells were counted from at least 5 fields per well with $20 \times$ magnification using inverted microscopy (Observer Z1; Carl Zeiss). The experiment was performed in triplicate and presented as percent cell adhesion \pm SEM.

\section{Statistical analysis}

Statistical analyses of cytopathic and cell-adhesion assays were performed using GraphPad Prism version 7.0 (GraphPad Software Inc., San Diego, CA, USA). Statistical significance of differences between groups was determined using a one-way ANOVA with Tukey's 
comparison of means as a post-hoc test. P-values $<0.05$ were considered significant. Results presented in graphs are mean values $\pm 95 \%$ confidence intervals for cell death plus standard errors of the mean for cell adhesion of at least 1000 cells per group/experiment and from three independent experiments.

\section{Results}

Mouse immunization with mixed Entamoeba species induced higher and faster antibody response than single species

Mouse immunization with E. moshkovskii trophozoite cells was shown to induce higher and faster totalantibody response than E. histolytica. Mice immunized with $E$. histolytica induced production of cross-Entamoeba species antibodies as E. moshkovskii (Fig. 1a, b). Compared to single species immunization, mice prechallenged with mixed trophozoite cells of E. histolytica and E. moshkovskii, followed by either E. histolytica or E. moshkovskii cells, induced significantly higher levels of total antibodies and specific IgG to both species (Fig. 1a-d). Only the mice pre-immunized with mixed $E$. moshkovskii and E. histolytica cells were able to stimulate cross-species IgG (Fig. 1c, d). Species-specific IgG antibodies of mice immunized with the single Entamoeba species decreased sharply to the same level as the preimmunized sera at 2 months after the last immunization. Levels of E. histolytica- and E. moshkovskii-specific IgG antibodies of mice immunized with mixed Entamoeba before individual species fell sharply at 2 months after the last boost, as seen in mice immunized with single Entamoeba species. However, these levels of E. histolytica- (Fig. 1c) and E. moshkovskii-specific IgG (Fig. 1d) remained about two-times higher than mice immunized with single Entamoeba species. Both E. histolytica and E. moshkovskii trophozoite cells induced a low level of cross-species IgA (Fig. 1e, f). Mice immunized with mixed Entamoeba species followed by E. histolytica stimulated the highest level of species-specific IgA; however, their levels decreased sharply after the last boost (Fig. 1e). Mice immunized with mixed Entamoeba species followed by E. moshkovskii stimulated a higher level of species-specific IgA than those immunized with $E$. moshkovskii cells alone (Fig. 1f). Furthermore, levels of $E$. moshkovskii-specific IgA antibodies remained high for at least 2 months after the last boost (Fig. 1f).

\section{Species-specific IgG antibodies promoted cytopathicity of E. histolytica toward $\mathrm{CHO}$ cells}

$\mathrm{CHO}$ cells were challenged with Entamoeba cells preincubated with serum collected at 2 weeks (B4) and 2 months (B5) after dose-4 of Entamoeba-cell immunizations. The cytopathic ability of Entamoeba cells is represented as percent death with 95\% $\mathrm{CI}$ of $\mathrm{CHO}$ cells. Entamoeba histolytica alone induced 33\% (95\% CI: 31.435.0\%) of CHO-cell death (Fig. 2a, PC), which was close (ANOVA: $\left.F_{(4,40)}=0.005, P=0.99\right)$ to those of amoeba cells incubated with pre-immunized serum $(33 \pm 2.7 \%)$. Entamoeba histolytica cells incubated with the B4 and B5 sera of mice immunized with heterologous Entamoeba species followed $E$. histolytica $(\mathrm{Eh}+\mathrm{Em}>\mathrm{Eh}$; ANOVA: $\left.F_{(2,24)}=19.64, P=0.01\right), E$. moshkovskii $(\mathrm{Eh}+\mathrm{Em}>\mathrm{Em}$; ANOVA: $\left.F_{(2,24)}=35.68, P=0.01\right)$ and the sera of $E$. histolytica (Eh; ANOVA: $F_{(2,20)}=7.53, P=0.01$ ) induced a significantly higher cytopathic ability of E. histolytica than cells pre-incubated with pre-immunized serum as well as the positive amoeba control without a serum (Fig 2a, cytopathic assay, E. histolytica). Numbers of $E$ histolytica cells $(68 \pm 2.2 \%)$ were found to adhere to $\mathrm{CHO}$ cells. Most E. histolytica cells pre-incubated with immunized sera, especially B5 serum of the mice immunized with $\mathrm{Eh}+\mathrm{Em}>\mathrm{Eh}$ cells, were found to promote to the parasite adhesion to CHO cells (ANOVA: $F_{(2,11)}=6.8, P=0.01$ ) (Fig. 2, E. histolytica) and Caco-2 cells (ANOVA: $F_{(5,16)}=13.86, P=0.01$ ) (Fig. 3a).

Interestingly, $E$. moshkovskii showed cytopathic activity of $15 \pm 3.4 \%$ (Fig. 2a, cytopathic assay, $E$. moshkovskii) and $88 \pm 1.8 \%$ adhesion to $\mathrm{CHO}$ cells (Fig. 2b, adhesion assay, E. moshkovskii). However, there was no difference in cytopathic ability (ANOVA: $\left.F_{(12,91)}=0.74, P=0.70\right)$ and $\mathrm{CHO}$ cell adhesion (ANOVA: $F_{(12,99)}=1.61, P=0.10$ ) of E. moshkovskii in serum pre-treated and un-treated conditions. Immunized sera were tested further for their effect on amoeba adhesion to Caco-2 cells. Despite inhibition of E. moshkovskii adhesion to Caco-2 cells, there was no significant difference (ANOVA: $F_{(5,30)}=1.91$, $P=0.12)$ in parasitic adhesion among the immunized, pre-immunized sera and serum un-treated amoeba (Fig. 3b).

\section{Cellular localization of mAb binding partners in $E$. moshkovskii and E. histolytica trophozoite cells}

Mice were immunized with either E. histolytica or $E$. moshkovskii trophozoite cells and boosted with a cell mixture of E. histolytica and E. moshkovskii (Eh $>\mathrm{Eh}+\mathrm{Em}$, and $E m>E h+E m$, respectively) to maximize the variety of Entamoeba-specific mAbs to study cellular localizations of the mAb-binding partners. Each set of immunized mice showed high antibody response to both $E$. histolytica and E. moshkovskii, respectively (Fig. 4a, b). In the immunization design with $E$. histolytica followed by mixed cells of $E$. histolytica and E. moshkovskii, $45 \%$ of mAbs were specific to E. histolytica (Eh), $34 \%$ to $E$. histolytica and E. dispar (Ehd), 9\% to pan-human Entamoeba; E. histolytica, E. moshkovskii and E. dispar (Ehmd), and 


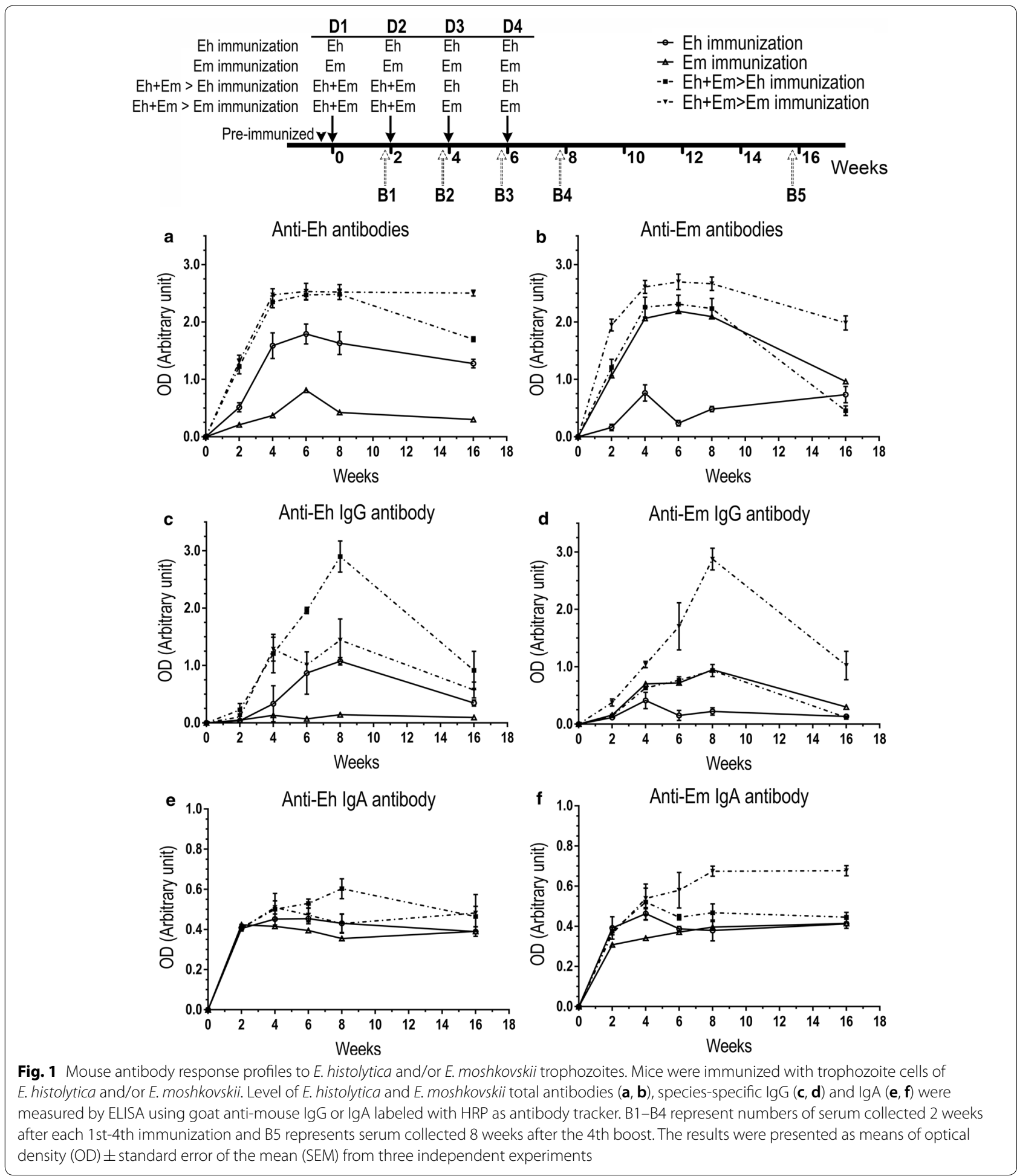

12\% to E. moshkovskii (Em) (Fig. 4a, Eh $>$ Eh + Em). On the other hand, the immunization design with E. moshkovskii followed by mixed Entamoeba cells resulted in 34\% of Em-specific mAb, 21\% of Ehd mAbs, 36\% of pan Ehmd
mAbs, and 9\% of Eh mAbs (Fig. 4b, Em > Eh + Em). These mAbs reacted against cytoplasmic, membranous and cytoskeletal fractions of Entamoeba cells. It was demonstrated that $78 \%$ of $E$. histolytica-specific mAbs targeted 

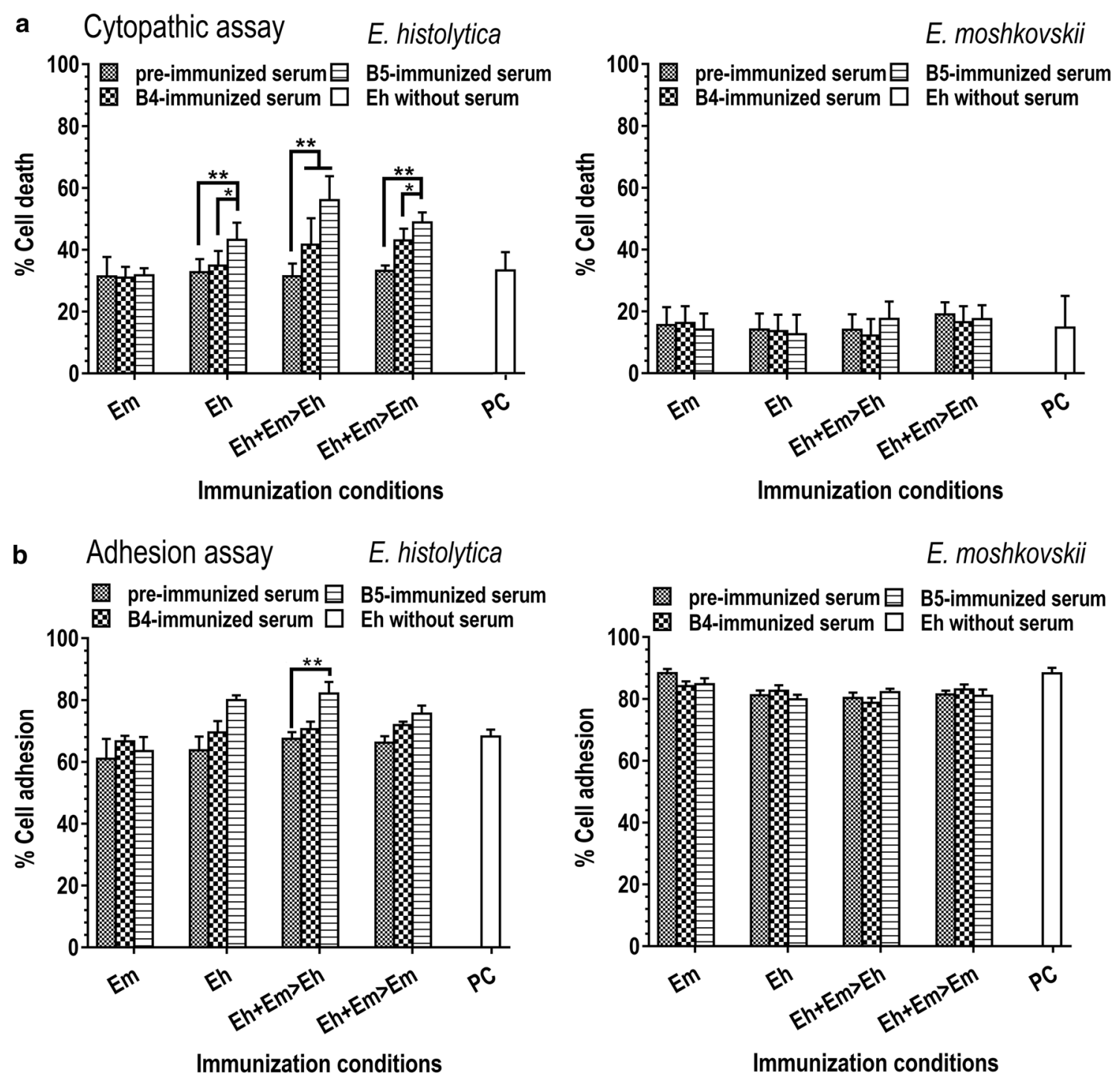

Immunization conditions

Fig. 2 Cytopathic and adhesion assays of E. histolytica and E. moshkovskii with mouse sera. CHO cells were incubated with E. histolytica and E. moshkovskii trophozoite cells in the presence of mouse sera immunized with E. histolytica (Eh) or E. moshkovskii (Em) alone, mixed of E. histolytica and E. moshkovskii followed by E. histolytica (Eh $+E m>E h)$, and the mixed cells followed by E. moshkovskii (Eh + Em $>$ Em). The effect of mouse sera on cytopathic activity and adhesion of E. histolytica and E. moshkovskii to $\mathrm{CHO}$ cells were determined by cell staining with propidium iodide (PI) and counting of $\mathrm{PI}$ positive and adhered $\mathrm{CHO}$ cells, respectively. $\mathrm{PC}$ is amoeba and $\mathrm{CHO}$ mixture without serum. B4 and B5 represent sera collected 2 weeks and after 4 times of trophozoite-cell immunization. Pre-immunized serum of each mouse was use as a negative control. The results are presented as percent cell death with 95\% confidence interval from three independent experiments. F-values refer to ANOVA across all three challenges, asterisks indicate significant differences between challenges (Tukey's post-hoc comparison), ${ }^{* *} P<0.01$ and ${ }^{*} P<0.05$

to cytoplasmic, $11 \%$ to cytoskeleton and $11 \%$ to membrane fractions (Fig. 4c). In contrast, $73 \%$ of E. moshkovskii-specific mAbs targeted membrane and only $27 \%$ toward cytoplasmic fractions. For cross-species mAbs, Ehm-specific mAbs equally targeted cytoplasm and membrane fractions of E. histolytica and E. moshkovskii trophozoite cells. Most of Ehd mAbs specifically directed to membrane (82\%) and $18 \%$ to cytoplasmic fractions of E. histolytica and E. dispar cells. About $69 \%$ of pan-Ehmd
mAbs were found to target the cytoplasm and $31 \%$ of them to cytoskeleton (Fig. 4c). An immunofluorescent assay was performed to confirm specificity and cellular localization of mAb-binding sites in Entamoeba trophozoite cells. Cells were directly reacted with a mAb to assess $\mathrm{mAb}$ binding at the outer membrane of the cells (non-permeabilized condition), while $\mathrm{mAb}$ access to plasma membrane and cytoplasmic components of the cells was performed through permeabilization using 
a

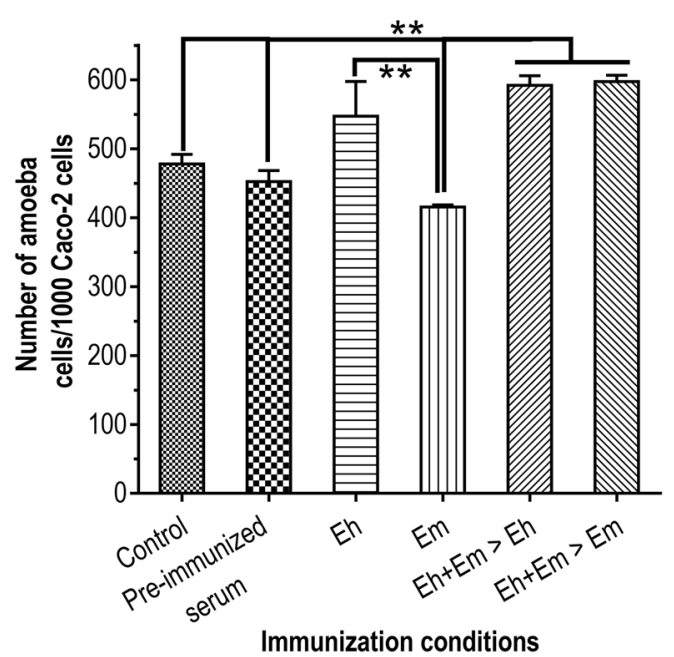

b

E. moshkovskii

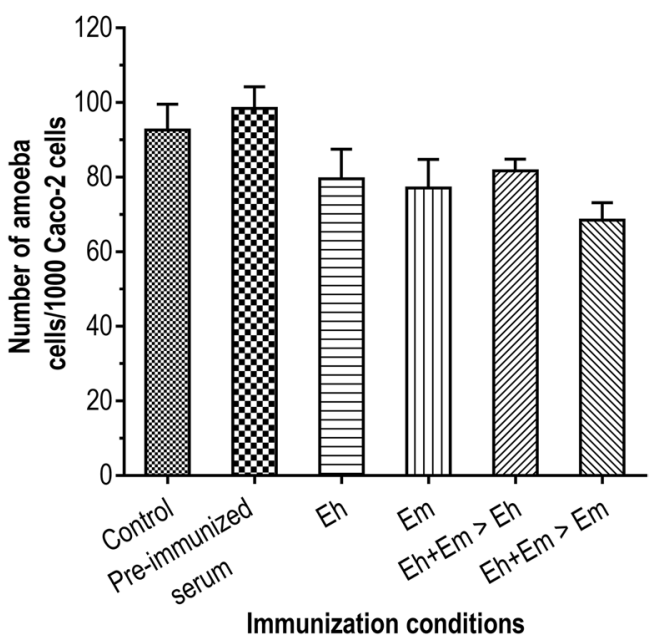

Fig. 3 Adhesion of E. histolytica and E. moshkovskii to Caco-2 cells. Trophozoite cells of E. histolytica (a) and E. moshkovskii (b) were incubated with the Caco-2 human epithelial cell line in the presence of a serum of mice immunized with trophozoite cells, with immunization conditions as mentioned and pre-immunized serum as a control. After washing, trophozoite cells bound on Caco-2 cells were counted and represented as means of amoeba number per 1000 Caco-2 cells \pm SEM from three independent experiments. Asterisks indicate significant differences between challenges, ${ }^{* *} P<0.01$. Abbreviation: SEM, standard error of the mean

triton X-100 detergent (permeabilized condition). All species-specific mAbs bound specifically to the corresponding Entamoeba species (Fig. 5) as shown in the previous result (Fig. 4b). Abundant binding sites of Eh mAb (Fig. 5, Anti-Eh4) and pan-Ehmd mAb (Fig. 5, AntiEhmd4) were observed in the cytoplasm of Entamoeba trophozoite cells. In contrast, most binding sites of Ehd $\mathrm{mAb}$ (Fig. 5, Anti-Ehd2) and Em mAb (Fig. 5, Anti-Em6) were at the outer cell membrane and cytoplasm. Binding localization of Ehm- (Fig. 5, Anti-Ehm2) and Ehd-specific mAbs (Fig. 5, Anti-Ehd2) spanned from outer- and innerplasma membrane and cytoplasm of the Entamoeba cells. In contrast, most binding partners of pan-human Ehmd $\mathrm{mAb}$ (Anti-Ehmd4) were associated with cytoplasmic components and were weakly detected at the outer membrane of the three Entamoeba species.

\section{Discussion}

Both humoral and cell-mediated immune responses have been demonstrated to play roles in E. histolytica infection and disease manifestation [22]. However, few details exist on host immune response to E. moshkovskii. In the present study, we found that E. moshkovskii trophozoites induced species-specific antibodies in mice faster than $E$. histolytica, suggesting a difference in host immune response to these closely related species. Furthermore, mice pre-challenged with mixed trophozoite cells of $E$. histolytica and E. moshkovskii followed by single Entamoeba species produced species-specific IgG and IgA antibodies to both invasive E. histolytica and non-invasive E. moshkovskii faster and higher than the single species immunizations. These high responses among Entamoeba-specific antibodies may explain the ability of people in endemic areas to tolerate Entamoeba infection, where mixed infections of Entamoeba species are always common [23, 24]. On the other hand, a high level or titer of E. histolytica-specific IgG has been widely used as a diagnostic key for amoebic liver abscess (ALA), a severe infection outcome of E. histolytica [25]. Here, upon intraperitoneal inoculation with Entamoeba trophozoite cells, we found that levels of E. histolytica and E. moshkovskiispecific IgG in mouse sera corresponded to doses/numbers of immunizations and dropped sharply after the last immunization. This result firstly reveals the association of a level of species-specific IgG of mice and the presence of these two Entamoeba species in the stomach. Measurement of serum IgG may be applied for monitoring intestinal infections by E. histolytica and E. moshkovskii trophozoites. Mice experienced with a E. histolytica and E. moshkovskii cell mixture prior to secondary immunization with either E. histolytica or E. moshkovskii, produced IgG antibodies to the latter species higher and faster than those mice immunized with single Entamoeba species. This may have resulted from the immunological memory of the mouse adaptive immune system [26]. IgA antibodies produced by plasma cells within the lamina propria serve as physical barrier to prevent intestinal mucus from adhesion and invasion of pathogens [27]. Antigens/ 


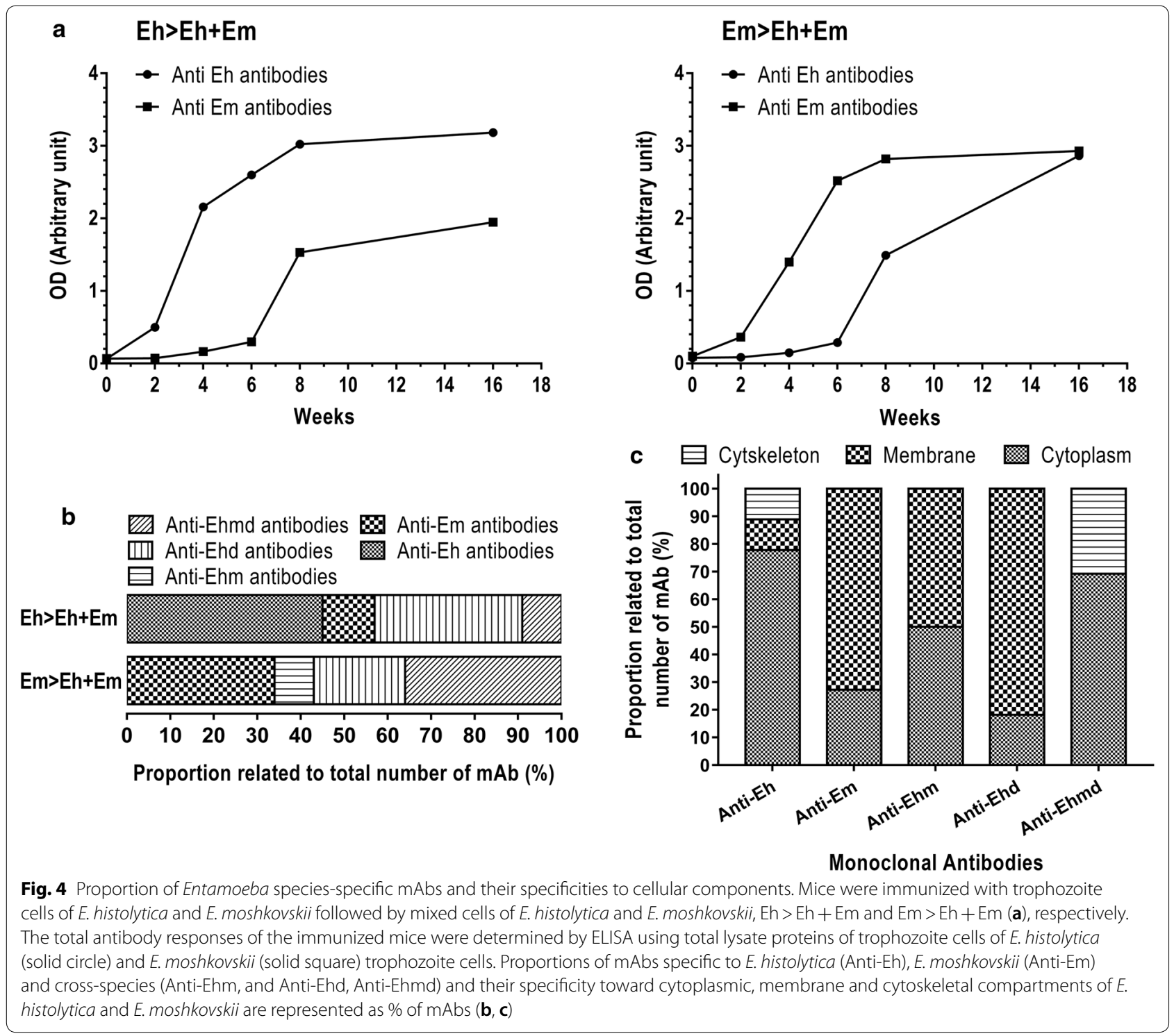

proteins of E. histolytica were injected via the oral, intradermal, intramuscular and subcutaneous routes together with adjuvants to elicit strong IgA antibody-, cell-mediated responses and protection of the host [28]. In this study, without any adjuvant, mixed E. histolytica and $E$. moshkovskii trophozoite cells followed by E. moshkovskii were inoculated in the intraperitoneal route, and could elicit IgA antibody response of mice more than the mixed E. histolytica and E. moshkovskii cells followed by E. histolytica or with E. moshkovskii alone. Furthermore, the $E$. moshkovskii-specific IgA antibodies were produced faster and lasted longer than those with E. histolytica. This may be due to the strong immunogenicity of E. moshkovskii and corresponds to previous reports that E. moshkovskii was eliminated from the host faster than $E$. histolytica $[17,29]$.
Pathogenicity of E. histolytica trophozoites is mediated by parasite adhesion with gut mucin through parasitic Gal/GalNAc lectin [30] and actions of cysteine proteinases as mentioned previously. IgG antibody responses of patients have been shown to associate with infection susceptibility and pathogenicity and re-infection of $E$. histolytica [22]. In this study, sera of mice immunized with $E$. histolytica inversely promoted cytopathicity of $E$. histolytica towards $\mathrm{CHO}$ cells. Most sera of the E. histolytica immunized mice were found to significantly enhance parasitic adhesion to colonic Caco-2 cells. However, mice immunized with E. histolytica and E. moshkovskii cells followed by E. histolytica promoted parasite adhesion to $\mathrm{CHO}$ cells. It was likely that increased in adhesion and cytopathic activity of E. histolytica to these cells are associated with a level of specific IgG antibodies in 


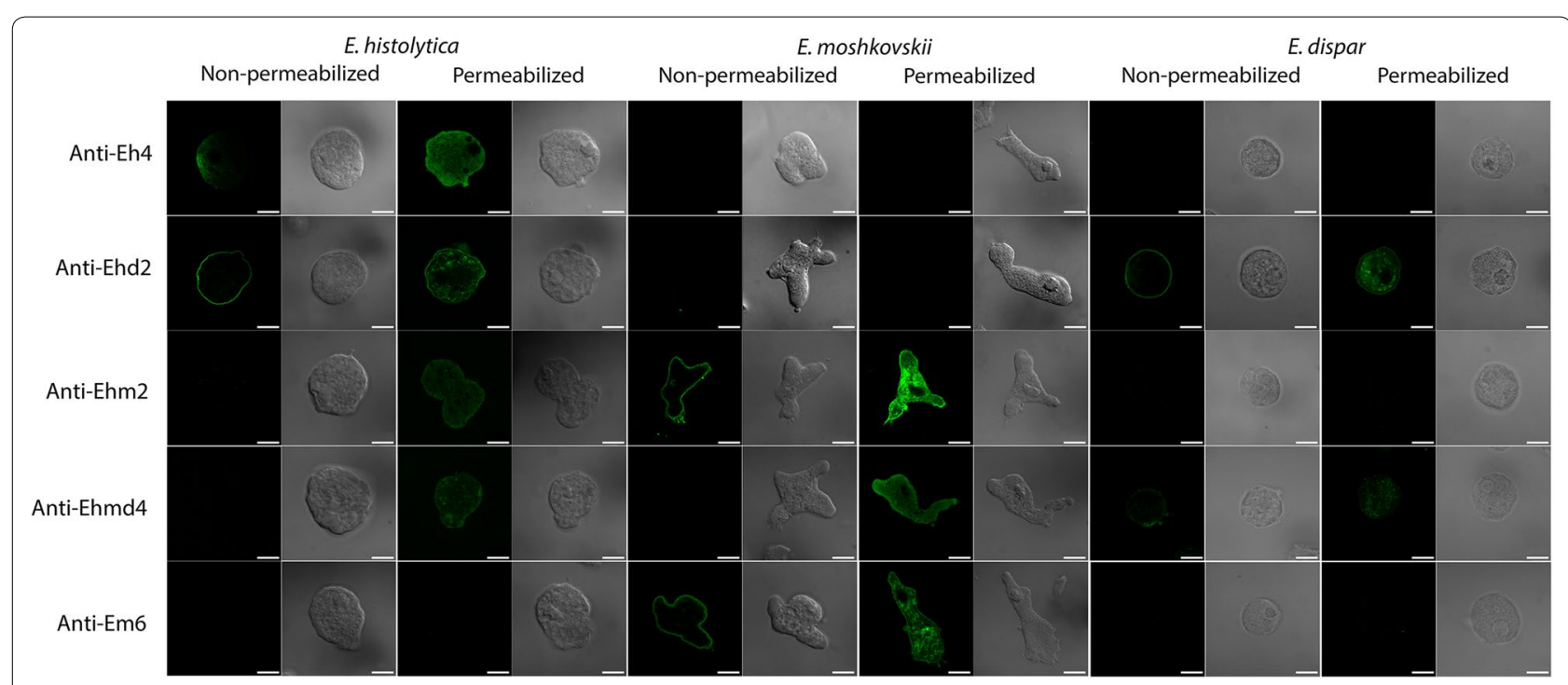

Fig. 5 Cellular localization of mAb-recognition molecules. Non-permeabilized or Triton X-100 permeabilized (0.2\%) trophozoite cells of E. histolytica, E. moshkovskii and E. dispar were stained with representative $m A$ bs by immunofluorescent assay. The bound mAbs were tracked by fluorescein isothyocyanate (FITC)-conjugated goat anti-mouse lgG antibodies and observed under confocal microscopy (LSM 700; Carl Zeiss). Scale-bars: 10 m

the immunized sera with the exception of B5-immunized sera. It should be noted that B5 sera were collected two months after the last immunization, where a level of $E$. histolytica specific IgG antibodies have decreased. Therefore, there remains no explanation for the positive effect of these sera on adhesion and cytopathic activity of the parasite. However, it might be due to the sum of affinity/avidity of polyclonal antibodies found in the immunized sera after repeated immunizations. The present study also reflects a difference in susceptibility of tissue cells for E. histolytica infection. In parasitic E. histolytica, the role of specific IgG antibodies in pathogenicity is not clear; however, it has been suggested to depend on IgG subclasses. IgG2 and IgG4 have been reported as markers to follow-up ALA and recurrent infection of the parasite, respectively, whereas IgG1 and IgG3 were found to associate with parasite clearance by immune cells $[25$, $31]$. Genetic background and family history have also been considered as factors contributing to difference in isotypes of E. histolytica specific IgG [25]. Antibodymediated pathogenicity and disease severity known as antibody-dependent enhancement (ADE) has been exclusively studied in dengue virus and found to occur during the secondary infection with different virus serotypes [32, 33]. During ADE, virus recognition antibodies promote virus entry into dendritic cells and macrophages through their Fc receptor and suppress host immune response, resulting in survival and high viral load and severe disease manifestation of the host [33, 34]. It is possible that $E$. histolytica-specific IgG might promote pathogenicity of E. histolytica through ADE. For E. moshkovskii-specific
IgG and IgA antibodies, despite having moderate to high antibody levels, there is no profound effect of the immunized sera on adhesion and pathogenicity of E. moshkovskii towards $\mathrm{CHO}$ cells as well as parasitic adhesion to human epithelial Caco-2 cells. It might imply an alternative host immune response and different pathogenicity of $E$. moshkovskii from its closely related species $E$. histolytica.

MAbs have been produced to E. moshkovskii and $E$. histolytica trophozoite cells and their characterizations revealed different localization of $\mathrm{mAb}$ binding targets in the virulent $E$. histolytica, non-virulent $E$. moshkovskii as well as non-pathogenic E. dispar. Most of the $E$. histolytica $\mathrm{mAbs}$ target to the cytoplasmic components, whereas most of the E. moshkovskii mAbs and cross-species (anti-Ehm and anti-Ehd2) and pan-human Entamoeba (anti-Ehmd) mAbs mostly target to the cell membrane. Although most E. histolytica-specific mAbs were shown to react to cytoplasm of the E. histolytica trophozoite, its membrane proteins, including Gal/GalNAc lectin, have been recognized by polyclonal antibodies in sera of immunized mice or humans. This would explain the enhancing effect of the immunized sera on host-cell adhesion and cytopathic abilities of the E. histolytica trophozoites. Compared to non-virulent $E$. moshkovskii, E. histolytica trophozoites might possess an evolutional design to conceal its surface molecules from the host immune system. It has been shown that E. histolytica possesses a thick and complicated cell surface containing lipopeptidophosphoglycan to protect the amoeba from the host complement system [35]. In addition, $E$. 
histolytica has been reported to modify its surface antigenic proteins in response to surrounding bacteria and growth conditions [36]. Furthermore, E. histolytica has been shown to cleave, translocate and release antibodysurface molecule complexes from the parasite cell surface by the parasite protease and actin rearrangement [37, 38]. These events were not observed in the non-pathogenic $E$. dispar [39]. Likewise, influenza virus causes reinfection by drifting its antigenic surface protein hemagglutinin, and further shifting of this protein also enables the virus to cause the cross-species infection [40, 41]. Modification of surface proteins in order to escape host immunity and prolong infection have also been reported in the sleeping sickness causative agent, Trypanosoma brucei, and the cattle protozoan parasite, Babesia bovis [42, 43].

Antigen variation is a crucial virulent feature that pathogens adopt to survive from the host immune system [44]. Here, we found that anti-Ehm $2 \mathrm{mAb}$ reacts to the inner membrane of $E$. histolytica trophozoite cells but is observed on the outer membrane of E. moshkovskii cells. Likewise, anti-Ehmd $4 \mathrm{mAb}$ binds to inner membrane and cytoplasmic components of E. histolytica and $E$. moshkovskii, trophozoites but is additional observed on the cell surface of non-pathogenic $E$. dispar. Therefore, it is likely that variation of $\mathrm{mAb}$ binding molecules might occur in these three closely related Entamoeba species. It has also been shown that $E$. histolytica has strain-specific antigens, which contribute to their virulence [45]. Plasmodium falciparum protozoans are the best model for altering its var gene family to escape from host immune recognition and prolong its infection [46].

\section{Conclusions}

A mixed infection of Entamoeba stimulated a higher IgA and IgG antibody response of the host compared to multi-infection by a single species of human-infecting Entamoeba. Entamoeba moshkovskii induced the host antibody response faster and higher than E. histolytica. Entamoeba-specific anti-sera promoted host cell adhesion and cytopathicity of E. histolytica, but showed no effect to those activities of E. moshkovskii. However, functional roles of the induced antibodies as well as levels of mucosal IgA in the mouse models with amoebic dysentery, colitis and liver abscess need to be further elucidated. Characterization of E. histolytica, E. moshkovskii, cross-species and pan-Entamoeba mAbs revealed profound surface antigen manipulation and translocation to conceal or escape from host immune recognition by $E$. histolytica over non-virulent E. moshkovskii and nonpathogenic $E$. dispar. These findings may be useful for further vaccine development and better understanding and pathogenicity and host immune modulation by $E$. histolytica in the future.

\section{Abbreviations}

MAb: monoclonal antibody; SDS-PAGE: sodium dodecyl sulfate-polyacrylamide gel electrophoresis; ELISA: enzyme-linked immunosorbent assay; HRP: horse radish peroxidase; $\mathrm{HCl}$ : hydrochloric acid; TMB: 3,3',5,5'-tetramethylbenzidine; EDTA: ethylene diamine tetraacetic acid; RPMI: Roswell Park Memorial Institute; PBS: phosphate-buffered saline; BSA: bovine serum albumin; CHO: Chinese hamster ovary; PI: propidium iodide; ALA: amoebic liver abscess; FITC: fluorescein isothiocyanate; ADE: antibody dependent enhancement; IL: interleukin; IFN- $\gamma$ : interferon gamma; TNF-a: tumor necrosis factor alpha.

\section{Acknowledgements \\ We would like to thank Professor Tomoyoshi Nozaki, Department of Biomedi- cal Chemistry, Graduate School of Medicine, The University of Tokyo, Japan, for his supervision and providing axenic Entamoeba trophozoite cells. Our sincere appreciation also goes to Professor Kris Chadee Department of Microbiol- ogy, Immunology and Infectious Diseases, University of Calgary, Canada, for providing the Caco-2 cell line. We would like to acknowledge Mr Irwin F. Chavez, Department of Tropical Hygiene, Faculty of Tropical Medicine, Mahidol University, Thailand, for reviewing the manuscript for grammar and syntax. \\ Funding \\ This work was supported by a grant from the Thailand Research Fund and Office of Higher Education Commission (grant no. MRG5480083) and Dean's Research Fund-2011, Faculty of Tropical Medicine, Mahidol University.}

\section{Availability of data and materials}

The data supporting the conclusions of this article are included within the article.

\section{Authors' contributions}

SM designed and planned the experiments and revised the manuscript. NK conducted the laboratory experiments, data analysis, and wrote the manuscript. SL conducted data analysis. ARP reviewed and edited the manuscript. All authors read and approved the final manuscript.

\section{Ethics approval and consent to participate}

The animal housing, immunization protocol and animal processing were approved by the Faculty of Tropical Medicine, Animal Care and Use Committee (permit number: FTM-ACUC 016/2012).

\section{Consent for publication}

Not applicable.

\section{Competing interests}

The authors declare that they have no competing interests.

\section{Publisher's Note}

Springer Nature remains neutral with regard to jurisdictional claims in published maps and institutional affiliations.

\section{Author details}

${ }^{1}$ Department of Protozoology, Faculty of Tropical Medicine, Mahidol University, Ratchawithi, Bangkok 10400, Thailand. ${ }^{2}$ Center for Vaccine Development, Institute of Molecular Biosciences, Mahidol University, Nakhorn-Pathom 73170, Thailand.

Received: 29 November 2018 Accepted: 26 February 2019

Published online: 13 March 2019

\section{References}

1. Wertheim H, Horby P, Woodall J. Amebiasis, Entamoeba histolytica. In: Wertheim H, Horby P, Woodall J, editors. Atlas of human infectious diseases. Oxford: Blackwell Publishing Ltd.; 2012. p. 127-8. 
2. Samie A, ElBakri A, AbuOdeh R. Amoebiasis in the tropics: epidemiology and pathogenesis. In: Rodriguez-Morales A, editor. Current topics in tropical medicine. Rijeka: InTech; 2012. p. 201-26.

3. Otto MP, Gerome P, Rapp C, Pavic M, Vitry T, Crevon L, et al. False-negative serologies in amebic liver abscess: report of two cases. J Travel Med. 2013;20:131-3.

4. Chalhoub S, Kanafani Z. Entamoeba histolytica pleuropulmonary infection. Case report and review of the literature. J Med Liban. 2012;60:122-4.

5. Viriyavejakul P, Riganti M. Undiagnosed amebic brain abscess. Southeast Asian J Trop Med Public Health. 2009;40:1183-7.

6. Tran VQ, Herdman DS, Torian BE, Reed SL. The neutral cysteine proteinase of Entamoeba histolytica degrades lgG and prevents its binding. J Infect Dis. 1998;177:508-11.

7. Garcia-Nieto RM, Rico-Mata R, Arias-Negrete S, Avila EE. Degradation of human secretory $\lg \mathrm{A} 1$ and $\lg \mathrm{A} 2$ by Entamoeba histolytica surface-associated proteolytic activity. Parasitol Int. 2008;57:417-23.

8. Huston CD, Houpt ER, Mann BJ, Hahn CS, Petri WA Jr. Caspase 3-dependent killing of host cells by the parasite Entamoeba histolytica. Cell Microbiol. 2000;2:617-25

9. Haque R, Huston CD, Hughes M, Houpt E, Petri WA Jr. Amebiasis. N Engl J Med. 2003;348:1565-73.

10. Wilson IW, Weedall GD, Hall N. Host-parasite interactions in Entamoeba histolytica and Entamoeba dispar: what have we learned from their genomes? Parasite Immunol. 2012;34:90-9.

11. Zhang Z, Mahajan S, Zhang X, Stanley SL Jr. Tumor necrosis factor alpha is a key mediator of gut inflammation seen in amebic colitis in human intestine in the SCID mouse-human intestinal xenograft model of disease. Infect Immun. 2003;71:5355-9.

12. Hamano S, Asgharpour A, Stroup SE, Wynn TA, Leiter EH, Houpt E. Resistance of C57BL/6 mice to amoebiasis is mediated by nonhemopoietic cells but requires hemopoietic IL-10 production. J Immunol. 2006;177:1208-13.

13. Clark CG, Diamond LS. The Laredo strain and other 'Entamoeba histolytica-like' amoebae are Entamoeba moshkovskii. Mol Biochem Parasitol. 1991;46:11-8.

14. Clark CG, Diamond LS. Differentiation of pathogenic Entamoeba histolytica from other intestinal protozoa by riboprinting. Arch Med Res. 1992:23:15-6.

15. Tanyuksel M, Ulukanligil M, Guclu Z, Araz E, Koru O, Petri WA Jr. Two cases of rarely recognized infection with Entamoeba moshkovskii. Am J Trop Med Hyg. 2007;76:723-4.

16. Shimokawa C, Kabir M, Taniuchi M, Mondal D, Kobayashi S, Ali IK, et al. Entamoeba moshkovskii is associated with diarrhea in infants and causes diarrhea and colitis in mice. J Infect Dis. 2012;206:744-51.

17. Shimokawa C, Senba M, Kobayashi S, Kikuchi M, Obi S, Olia A, et al. Intestinal inflammation-mediated clearance of amebic parasites is dependent on IFN-gamma. J Immunol. 2018;200:1101-9.

18. Sri-Hidajati B, Basuki S, Pusarawati S, Kusmartisnawati K, Rossyanti L, Sulistyowati SW, et al. Comparison of multiplex single round PCR and microscopy in diagnosis of amoebiasis. Afr J Infect Dis. 2018;12:120-6.

19. Moonsom S, Khunkeawla P, Kasinrerk W. Production of polyclonal and monoclonal antibodies against CD54 molecules by intrasplenic immunization of plasmid DNA encoding CD54 protein. Immunol Lett. 2001;76:25-30.

20. Ralston KS, Solga MD, Mackey-Lawrence NM, Somlata, Bhattacharya A Petri WA. Trogocytosis by Entamoeba histolytica contributes to cell killing and tissue invasion. Nature. 2014;508:526-30.

21. Teixeira JE, Sateriale A, Bessoff KE, Huston CD. Control of Entamoeba histolytica adherence involves metallosurface protease 1, an M8 family surface metalloprotease with homology to leishmanolysin. Infect Immun. 2012;80:2165-76.

22. Haque R, Duggal P, Ali IM, Hossain MB, Mondal D, Sack RB, et al. Innate and acquired resistance to amebiasis in Bangladeshi children. J Infect Dis. 2002;186:547-52.

23. Anuar TS, Al-Mekhlafi HM, Abdul Ghani MK, Azreen SN, Salleh FM, Ghazali $\mathrm{N}$, et al. Different clinical outcomes of Entamoeba histolytica in Malaysia: does genetic diversity exist? Korean J Parasitol. 2013;51:231-6.
24. Al-Areeqi MA, Sady H, Al-Mekhlafi HM, Anuar TS, Al-Adhroey AH, Atroosh WM, et al. First molecular epidemiology of Entamoeba histolytica, E. dispar and E. moshkovskii infections in Yemen: different species-specific associated risk factors. Trop Med Int Health. 2017;22:493-504.

25. Kaur U, Sharma AK, Sharma M, Vohra H. Distribution of Entamoeba histoIytica Gal/GalNAc lectin-specific antibody response in an endemic area. Scand J Immunol. 2004;60:524-8.

26. MacLeod MK, Kappler JW, Marrack P. Memory CD4 T cells: generation, reactivation and re-assignment. Immunology. 2010;130:10-5.

27. Lamm ME. Current concepts in mucosal immunity. IV. How epithelial transport of IgA antibodies relates to host defense. Am J Physiol. 1998;274:G614-7.

28. Quach J, St-Pierre J, Chadee K. The future for vaccine development against Entamoeba histolytica. Hum Vaccines Immunother. 2014;10:1514-21.

29. Shimokawa C, Culleton R, Imai T, Suzue K, Hirai M, Taniguchi T, et al. Species-specific immunity induced by infection with Entamoeba histolytica and Entamoeba moshkovskii in mice. PLoS One. 2013;8:e82025.

30. Chadee K, Petri WA Jr, Innes DJ, Ravdin JI. Rat and human colonic mucins bind to and inhibit adherence lectin of Entamoeba histolytica. J Clin Invest. 1987;80:1245-54.

31. Bernin H, Marggraff C, Jacobs T, Brattig N, Le VA, Blessmann J, et al. Immune markers characteristic for asymptomatically infected and diseased Entamoeba histolytica individuals and their relation to sex. BMC Infect Dis. 2014;14:621.

32. Gan ES, Ting DH, Chan KR. The mechanistic role of antibodies to dengue virus in protection and disease pathogenesis. Expert Rev Anti Infect Ther. 2017;15:111-9.

33. Ubol S, Phuklia W, Kalayanarooj S, Modhiran N. Mechanisms of immune evasion induced by a complex of dengue virus and preexisting enhancing antibodies. J Infect Dis. 2010;201:923-35.

34. Flipse J, Wilschut J, Smit JM. Molecular mechanisms involved in antibodydependent enhancement of dengue virus infection in humans. Traffic. 2013;14:25-35

35. Nakada-Tsukui K, Nozaki T. Immune response of amebiasis and immune evasion by Entamoeba histolytica. Front Immunol. 2016;7:175.

36. Bhattacharya A, Ghildyal R, Prasad J, Bhattacharya S, Diamond LS. Modulation of a surface antigen of Entamoeba histolytica in response to bacteria. Infect Immun. 1992;60:1711-3.

37. Baxt LA, Baker RP, Singh U, Urban S. An Entamoeba histolytica rhomboid protease with atypical specificity cleaves a surface lectin involved in phagocytosis and immune evasion. Genes Dev. 2008;22:1636-46.

38. Chavez-Munguia B, Talamas-Rohana P, Castanon G, Salazar-Villatoro L, Hernandez-Ramirez V, Martinez-Palomo A. Differences in cap formation between invasive Entamoeba histolytica and non-invasive Entamoeba dispar. Parasitol Res. 2012;111:215-21.

39. Calderon J, de Lourdes Munoz M, Acosta HM. Surface redistribution and release of antibody-induced caps in entamoebae. J Exp Med. 1980:151:184-93.

40. Das SR, Hensley SE, Ince WL, Brooke CB, Subba A, Delboy MG, et al. Defining influenza A virus hemagglutinin antigenic drift by sequential monoclonal antibody selection. Cell Host Microbe. 2013;13:314-23.

41. Shimizu K. Mechanisms of antigenic variation in influenza virus. Nihon Rinsho. 2000;58:2199-205.

42. Horn D. Antigenic variation in African trypanosomes. Mol Biochem Parasitol. 2014;195:123-9.

43. Dzikowski R, Deitsch K. Antigenic variation by protozoan parasites: insights from Babesia bovis. Mol Microbiol. 2006;59:364-6.

44. Rosen F, Weiss R. Pathogens have evolved various means of evading or subverting normal host defenses. In: Janeway Jr C, Travers P, Walport M, Shlomchik M, editors. Immunobiology: the immune system in health and disease. 5th ed. New York: Garland Science; 2001.

45. Ishaq M, Padma MC. Antigenic variations among strains of Entamoeba histolytica. Ann Trop Med Parasitol. 1980;74:373-5.

46. Recker M, Buckee CO, Serazin A, Kyes S, Pinches R, Christodoulou Z, et al. Antigenic variation in Plasmodium falciparum malaria involves a highly structured switching pattern. PLoS Pathog. 2011;7:e1001306. 